\title{
The eighth edition TNM stage classification for thymic tumors: What do I need to know?
}

\author{
Usman Ahmad, MD, FACS
}

Feature Editor's Introduction-Thymic epithelial tumors (TETs) are rare, enigmatic tumors. The incidence of thymoma is 1.5 cases per 1 million individuals, and thymic carcinomas are even less frequent. Thymomas are the most common tumors of the anterior mediastinum, however, and are routinely encountered in the clinics of thoracic surgeons. Consequently, thought leaders in thoracic surgery have been driving forces in the field of TETs. This is particularly true for staging, and we are in the midst of a paradigm shift from the traditional Masaoka-Koga system to a TNM system. The rarity of TETs is associated with obvious challenges in the development and refinement of their staging systems, and the unique behavior of TETs necessitates unique considerations. For example, the dominant mode of spread of TETs is local, and thymomas rarely disseminate through the lymphatic system. As a result, our staging systems are traditionally derived from surgical cohorts, and special consideration has recently been directed at $N$ staging descriptors. Importantly, the 5year survival of patients with thymoma is approximately $90 \%$ and extended time periods are required to accurately measure stage-specific outcomes. To overcome these challenges, a historic international partnership was formed between the International Association for the Study of Lung Cancer and organizations including the International Thymic Malignancies Interest Group, the European Society of Thoracic Surgeons, the Chinese Alliance for Research on Thymomas, and the Japanese Association of Research in Thymus. A collective database of nearly 10,000 cases was created to develop a TNM staging system for TETs that has since been adopted by the American Joint Committee on Cancer and the Union for International Cancer Control and that is included in the eighth edition of the TNM classification of malignant tumors. It is anticipated that this TNM system will result in more accurate staging for patients with TETs; however, its adoption into practice will require active participation and

From the Department of Cardiothoracic Surgery, Heart, Vascular, and Thoracic Institute; Transplant Institute; and Tausig Cancer Institute, Cleveland Clinic, Cleveland, Ohio.

Received for publication May 26, 2020; revisions received Oct 1, 2020; accepted for publication Oct 7, 2020; available ahead of print Nov 13, 2020.

Address for reprints: Usman Ahmad, MD, FACS, Department of Cardiothoracic Surgery, Heart and Vascular Institute, Cleveland Clinic, 9500 Euclid Ave, Cleveland, OH 44195 (E-mail: ahmadu@ @cf.org).

J Thorac Cardiovasc Surg 2021;161:1524-9 0022-5223/ $\$ 36.00$

Copyright $₫ 2021$ Published by Elsevier Inc. on behalf of The American Association for Thoracic Surgery

https://doi.org/10.1016/j.jtcvs.2020.10.131

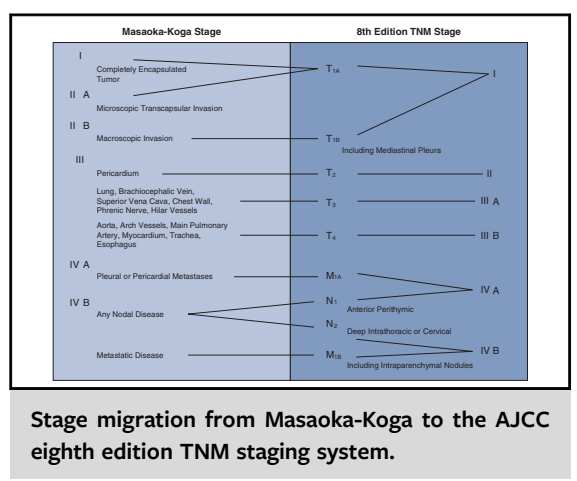

CENTRAL MESSAGE

The TNM staging system for thymic malignancies is based on international data and expert input. Barriers to widespread adoption need to be explored as we move toward future iterations.

This Invited Expert Opinion provides a perspec tive on the following paper: J Thorac Oncol. 2020 Mar;15(3):436-447 jtho.2019.11.013. Epub 2019 Nov 27.

See Commentary on page 1530.

rigor. First and foremost, it will require understanding. To that end, a clear and concise instrument for this is offered in the following Invited Expert Opinion article.

\section{Bryan M. Burt, MD}

Modern oncologic decision making relies on accurate clinical and pathologic staging. Appropriate stage assignment is key in choice of therapy, prognostication, studying response, and reporting outcomes. Thymic epithelial tumors hold a unique place among thoracic malignancies due to their relative rarity, often indolent course, and propensity for local and not systemic progression. ${ }^{1}$

Until recently, our knowledge of disease biology and clinical management was entirely based on multiple small institutional series. Not surprisingly, 15 unique staging classifications had been proposed for thymic neoplasms. ${ }^{2}$ 
TABLE 1. T, $N$, and $M$ definitions in the American Joint Committee on Cancer eighth edition cancer staging system

\begin{tabular}{cl}
\hline Category & \multicolumn{1}{c}{ Definition* } \\
\hline T1 & $\begin{array}{c}\text { Encapsulated or unencapsulated, with or without extension } \\
\text { into mediastinal fat } \\
\text { Extension into mediastinal pleura }\end{array}$ \\
b & Pericardium \\
T2 & Lung, brachiocephalic vein, superior vena cava, chest wall, \\
T3 & phrenic nerve, hilar (extrapericardial) pulmonary vessels \\
& Aorta, arch vessels, main pulmonary artery, myocardium, \\
T4 & trachea, or esophagus \\
& No nodal involvement \\
N0 & Anterior (perithymic) nodes \\
N1 & Deep intrathoracic or cervical nodes \\
N2 & No metastatic pleural, pericardial, or distant sites \\
M0 & \\
M1 & Separate pleural or pericardial nodule(s) \\
a & Pulmonary intraparenchymal nodule or distant organ \\
b & metastasis \\
\hline
\end{tabular}

*Definition is involvement of these elements.

Significant variability existed among these staging systems and the need for a comprehensive and consistent system was obvious. The need for an evidence-based, robust staging system is highlighted by the widely adopted MasaokaKoga system, which was derived from a retrospective series of just 96 patients.

An international collaboration between the International Thymic Malignancy Interest Group (ITMIG), European Society of Thoracic Surgeons, Japanese Association of Research in Thymus, and the Chinese Alliance for Research on Thymomas renewed enthusiasm in the study of thymic tumors and resulted in a retrospective database of approximately 10,000 cases. ${ }^{4}$ In partnership with the International Association for the Study of Lung Cancer (IASLC), these

TABLE 2. Stage categorization of thymic malignancy TNM staging system

\begin{tabular}{lllll}
\hline T/M & Subcategory & N0 & N1 & N2 \\
\hline T1 & 1a & I & IVa & IVb \\
& 1b & & & \\
T2 & & II & IVa & IVb \\
T3 & & IIIa & IVa & IVb \\
T4 & & IIIb & IVa & IVb \\
M1 & a & IVa & IVa & IVb \\
& b & IVb & IVb & IVb \\
\hline
\end{tabular}

data were used to develop a TNM stage classification (Table 1 ), ${ }^{5}$ which was adopted by the American Joint Committee on Cancer and the Union for International Cancer Control as part of the eighth edition of the TNM classification of malignant tumors (Table 2).

Thoracic surgeons, trainees, and data managers should have a clear understanding of key features and limitations of the staging system to guide clinical decision making and further research. These issues are germane to the everyday practice of thymic tumor oncology. This article highlights areas of controversy and potential barriers to adoption of the new TNM-based system.

\section{WHAT DO I NEED TO KNOW ABOUT THE DATA USED TO GUIDE THE PROPOSAL?}

Retrospective data were contributed by participating organizations and was analyzed by the Cancer Research and Biostatistics group. The data represent contributions from 105 institutions. Although patient treatment dates ranged from the 1950 s to 2014 , anyone treated before 1990 was excluded from analysis. ${ }^{6}$ Details of radiographic and surgical modalities used for clinical staging were not collected. Although positron emission tomography has become standard of care for patients with other thoracic malignancies, its use in thymic tumor staging is not widely adopted.

The majority of data were provided by participating pathologists and surgeons. Breakdown of treatment modalities reveals that the data submitted was skewed toward resectable disease. Less than $1 \%$ of patients had no treatment. Treatment details were available in $77 \%$ of cases, in which surgery alone $(40 \%)$ or in combination with chemotherapy $(5 \%)$, radiotherapy $(19 \%)$, or chemoradiotherapy $(12 \%)$ accounted for the predominant treatment modality. Less than $1 \%$ of patients had nonsurgical treatment. ${ }^{6}$ Nodal sampling and its reporting has been a routine practice primarily from Japanese contributors. ${ }^{7,8}$ Analyses of nodal metastases and staging criteria relied heavily on Japanese data.

\section{HOW ARE STAGE GROUPINGS ASSIGNED AND WHY SHOULD I CARE?}

For clinicians involved in the practice of oncology, it is important to understand the terminology of staging because it forms the basis of documenting and communicating extent of disease and, potentially, rationale for choice of treatment. Staging efforts are governed by the American Joint Committee on Cancer and Union for International Cancer Control and are updated on a periodic basis. Thoracic tumor stage recommendations are made by the IASLC Staging and Prognostication Factors Committee.

In general, primary tumor characteristics, number and/or location of involved lymph nodes, pleural or pericardial disease, and presence of distant metastases are examined to identify factors that predict recurrence of disease and 
overall survival. After identification of individual T, N, and $M$ prognostic factors, combinations are created and assigned stage groupings such that increasing stage identifies individuals with higher risk of recurrence and lower overall survival. In an ideal world, these decisions would be based on reliably collected, uniformly reported data across the spectrum of tumor progression. However, in the real world, most of these decisions are based on retrospective data (more commonly reported in patients who undergo treatment) and is inevitably biased. Therefore, it is not uncommon for some stage classifications to be assigned based on expert consensus only. That is partly why after publication of every new edition of stage classification, it is customary to see multiple publications showing varying predictive ability of the model using patient data from different parts of the world.

This TNM system is based on the largest body of thymic tumor data that has ever been analyzed. There are several prominent features that differentiate the new system. Foremost is the breakdown of stages in to T, N, and M categories, whereas only stages were available in the MasaokaKoga system. Although the ultimate stage assigned may be the same, the breakdown allows detailed reporting of the extent of disease. Capsular and mediastinal pleural involvement are considered part of T1 and, in the absence of nodal disease, constitute stage I. This stage downshift will lead to less use of adjuvant radiation therapy in completely resected tumors. Pericardial invasion was assigned stage III in Masaoka-Koga; however, is assigned T2-stage II in TNM. Direct local invasion is categorized into T3/T4stage IIIa/b. Nodal involvement was assigned stage IVb in Masaoka-Koga has been downgraded to IVa in TNM. Pleural and pericardial nodules are considered M1a and assigned stage IVa, which is similar to the Masaoka-Koga system. Parenchymal nodules and distant metastases remain assigned to stage IVb.

With methodical documentation of $\mathrm{T}, \mathrm{N}$, and $\mathrm{M}$ components, it is expected that the TNM system will allow better capture of these stage components. This particular iteration is based on retrospectively collected data that has significant gaps. With more complete documentation of the TNM characteristics, future iterations will allow even more clear differentiation of stages and prognostic variables. This may in turn allow improved assessment of treatment modalities and surveillance routines.

\section{WHAT ARE THE LIMITATIONS OF THIS STAGING SYSTEM?}

Although the eighth edition thymic tumor staging represents the most robust effort to date, several caveats rooted in source of data; quality of reporting; and most importantly, variability in practice, influence the strength of this tool.
For thymic tumor stage groupings, the majority of the classifications are based on actual data analysis. However, as noted above, very few patients had nonsurgical therapy and therefore the staging system likely loses some predictive ability toward the higher end of the disease spectrum. The nodal map was based on Japanese practice patterns with boundaries of N1 and N2 nodal stations determined empirically with some consideration of feasibility of surgical sampling. ${ }^{8}$ Differentiation between pleural/pericardial nodules (M1a) and parenchymal nodules (M1b) could not be elucidated statistically due to paucity of data in these categories and the differentiation was made empirically. Although the staging system may perform well in its predictive ability in early-stage resectable tumors, it is unlikely to have similar robustness in patients with unresectable disease or those who do not undergo treatment.

\section{UNIQUE FEATURES OF THE EIGHTH EDITION THYMIC TNM CLASSIFICATION}

The stage classification is based on anatomic extent of disease progression and therefore reflects on resectability rather than true disease biology. This is supported by the fact that thymic tumors are more likely to progress and invade locally, and systemic metastases are less common even in the presence of advanced local disease. In addition, as mentioned above, a surgery-heavy data set was used to guide the staging criteria.

In comparison with the popular Masaoka-Koga system (Figure 1), capsular invasion was not noted to have prognostic significance and was omitted. Similarly, involvement of mediastinal pleura had no significance except in Japanese data; therefore, $\mathrm{T} 1 \mathrm{a}$ and $\mathrm{T} 1 \mathrm{~b}$ categories were created. Although any invasion across the tumor capsule rendered it stage II in the Masaoka-Koga system, transcapsular invasion, including that into mediastinal pleura, is now categorized into stage I. At such level of invasion, adjuvant radiation therapy had been a consideration for Masaoka-Koga stage II thymomas. Now that local invasion up to mediastinal pleura is downstaged to stage I, this will influence decision making for adjuvant radiation therapy. It is not surprising that most previous data showed no benefit of adjuvant radiation therapy in Masaoka-Koga stage II thymomas. ${ }^{9}$

Local invasion into surrounding structures, including pericardium, innominate vein, superior vena cava, and major arterial conduits is the hallmark of locally advanced thymic tumors. The $\mathrm{T}$ stage had to incorporate invasion into these structures while also taking into account the relative clinical significance, multiple areas of involvement, and resectability. This led to creation of a tiered system designated by the most critical structure involved. Concomitant involvement of any other structure below the 
Masaoka-Koga Stage

\section{8th Edition TNM Stage}

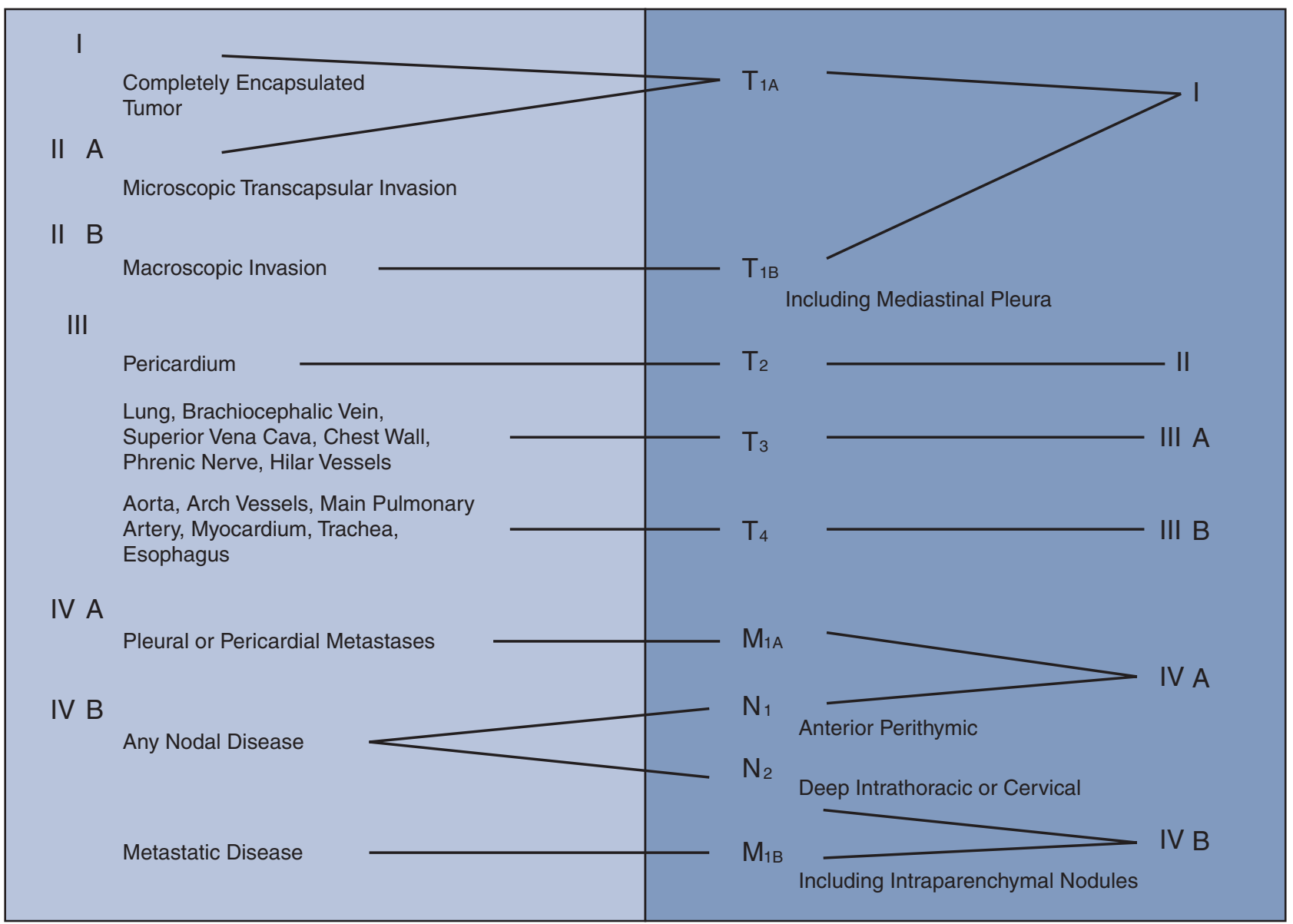

FIGURE 1. Stage migration from Masaoka-Koga to American Joint Commission on Cancer eighth edition TNM system. TNM, Traditional Masaoka-Koga system.

highest tier did not change the $\mathrm{T}$ stage. Tumor size had no prognostic value.

The nodal map is empiric and is based mostly on proximity of the tumor to the nodes. Nodal involvement was noted in $2 \%$ of thymomas, $27 \%$ of thymic carcinomas, and $28 \%$ of neuroendocrine tumors. The vast majority of involved nodes were in the parathymic anterior. Only $26 \%$ of thymomas that had lymph node involvement, $30 \%$ of thymic carcinoma, and $45 \%$ of neuroendocrine tumors of the thymus had $\mathrm{N} 2$ or middle mediastinal nodal involvement. ${ }^{8}$ Extent of nodal dissection and details of sampled stations cannot be assessed due to the nature of the data.

Due to small number of patients, the true significance of various levels of nodal involvement is not as clear-cut as it is in other thoracic malignancies. Ultimately, 2 nodal zones were determined based on proximity to anterior mediastinum, extrapolation from Japanese experience, and surgical ease of sampling anterior mediastinal nodes. The ITMIG had previously provided expert consensus based guidance on which N1 and N2 stations should be routinely sampled. ${ }^{10}$

Because the TNM system represents anatomic extent of disease, histology was not taken into consideration despite difference in survival and cumulative incidence of recurrence. For example, the 5-year and 10-year overall survival for completely resected stage I thymoma was $95 \%$ and $88 \%$, respectively, whereas similarly treated thymic carcinoma imparted 5-year and 10-year overall survival of $84 \%$ and $72 \%$, respectively. ${ }^{5}$

Whether staging systems should incorporate tumorrelated factors outside of anatomic extent of disease to help increase the ability to predict recurrence and survival and hence guide therapy remains a contentious issue. Certainly esophageal cancer staging ${ }^{11}$ has moved in that direction, whereas lung cancer staging, which is by far the 
most data driven, assigns stage based on extent of disease. ${ }^{12}$ It is important to note that the $\mathrm{T}, \mathrm{N}$, and $\mathrm{M}$ components of a tumor are not independent of each other. In clinical practice, a higher $\mathrm{T}$ stage could increase the pretest probability of a greater $\mathrm{N}$ and $\mathrm{M}$ stage; hence, changing the investigational staging strategy.

If the field moves toward using TNM data as part of prognostic modeling, it will have to be incorporated in to a larger grid that includes patient demographic characteristics, comorbidities, tumor histology, and response to therapy.

\section{WHAT ARE THE CHALLENGES IN ADOPTION AND INTEGRATION?}

With its implementation in January 2018, the TNM system required a paradigm shift in documentation of clinical staging, intraoperative nodal assessment, and pathologic reporting of specimens. To assess the adoption and to identify potential rate limiting steps, the IASLC Thymic Domain Staging and Prognostication Factors Committee undertook an international web-based survey. ${ }^{13}$ Members of various thymic and thoracic communities were approached and invited to answer 50 questions related to various aspects of staging modalities, utilization of the staging system, and treatment modalities. Significant differences were noted in the perceived importance of various $\mathrm{T}$ categories and reported rates of nodal sampling.

Computed tomography was the most commonly employed imaging modality for clinical staging with significantly lesser use of positron-emission tomography and magnetic resonance imaging. Only half the responders found the nodal map to be helpful. A similar percentage indicated performing $\mathrm{N} 1$ nodal sampling during surgery, whereas only $20 \%$ indicated performing N2 sampling for thymomas and $41 \%$ for thymic carcinomas. A higher $\mathrm{T}$ stage was more likely to drive nodal sampling. Nodal sampling, especially $\mathrm{N} 2$, sampling was less commonly reported with minimally invasive surgeries. Forty percent of responders reported only removing the lymph nodes that came out with the main specimen in minimally invasive operations. In general, more lymph node sampling was reported for thymic carcinomas.

The use of TNM system was more common in Asia and so was the routine systematic nodal sampling compared with North America. Significant use of the Masaoka-Koga system was still reported. Although the TNM system is here to stay and will likely continue to be improved with subsequent iterations, adoption and complete adherence will take time.

Nodal involvement was part of the staging in MasaokaKoga system, but routine systematic sampling was not commonly discussed. Routine nodal sampling has been part of practice in Japan and China but not so much in
European and North American centers. Now that there is a category of $\mathrm{N}$ status that needs to be adjudicated for the case, more attention to nodal sampling and staging can be expected.

There has been a trend toward increasing use of minimally invasive approaches for early-stage tumors. ${ }^{14}$ In this context, it is important to reiterate that the ability to perform complete (ie, R0) resection should not be compromised by the surgical approach. A clinical T1 N0 M0 (stage I) tumor could now be large and invade mediastinal pleural; therefore, the choice and decision of surgical approach should take these factors in to account and should be optimized to achieve complete resection.

Similarly, appropriate nodal sampling should not be compromised by the surgical approach. The rate of nodal metastases in thymomas is low, and in a majority of cases, the involved nodes are in the anterior mediastinum. Sampling these N1 nodes remains feasible through minimally invasive surgical approaches. Thymic carcinoma is more likely to have N2 nodal metastases and appropriate sampling of N1 and N2 nodes is important in appropriately staging these tumors.

\section{WHAT'S NEXT? WHERE TO?}

The TNM staging system is a significant step forward in the study and care of thymic tumors. The most remarkable achievement is the ability to uniformly report tumor characteristics through a consistent staging system utilized throughout the world. Although this system was based on historical retrospective data, prospective data collection is already underway by Chinese and European groups and the ITMIG. Analysis of more reliably collected staging data that is based on contemporary imaging and treatment modalities will likely result in further refinement of the staging system. Now that uniform stage reporting is underway, we can move on to studying the variability in treatment paradigms and work toward improving clinical outcomes and quality of life of these patients.

\section{Conflict of Interest Statement}

Dr Ahmad is an advisory board member of International Association for the Study of Lung Cancer-Staging and Prognostication Factors Committee (Thymic Domain), has received grant funding from International Association for the Study of Lung Cancer-Staging and Prognostication Factors Committee for work related to TNM staging of thymic malignancies, and is a member of International Thymic Malignancy Interest Group database and steering committees.

The Journal policy requires editors and reviewers to disclose conflicts of interest and to decline handling or reviewing manuscripts for which they may have a conflict 
of interest. The editors and reviewers of this article have no conflicts of interest.

\section{References}

1. Ahmad U, Huang J. Thymic tumors. In: LoCicero J, Feins R, Rocco G, Colcon Y, Meyers B. Shield's General Thoracic Surgery. 8th ed. Philadelphia, PA: Lipppincott Williams \& Wilkins; 2018.

2. Filosso PL, Ruffini E, Lausi PO, Lucchi M, Oliaro A, Detterbeck F. Historical perspectives: the evolution of the thymic epithelial tumors staging system. Lung Cancer. 2014;83:126-32.

3. Masaoka A, Monden Y, Nakahara K, Tanioka T. Follow-up study of thymomas with special reference to their clinical stages. Cancer. 1981;48: 2485-92.

4. Huang J, Ahmad U, Antonicelli A, Catlin AC, Fang W, Gomez D, et al. Development of the international thymic malignancy interest group international database: an unprecedented resource for the study of a rare group of tumors [published correction appears in J Thorac Oncol. 2014;9:1804]. J Thorac Oncol. 2014;9:1573-8.

5. Detterbeck FC, Stratton K, Giroux D, Asamura H, Crowley J, Falkson C, et al. The IASLC/ITMIG thymic epithelial tumors staging project: proposal for an evidence-based stage classification system for the forthcoming (8th) edition of the TNM classification of malignant tumors. J Thorac Oncol. 2014;9(9 Suppl 2):S65-72.

6. Nicholson AG, Detterbeck FC, Marino M, Kim J, Stratton K, Giroux D, et al. The IASLC/ITMIG thymic epithelial tumors staging project: proposals for the T Component for the forthcoming (8th) edition of the TNM classification of malignant tumors. J Thorac Oncol. 2014;9(9 Suppl 2):S73-80.

7. Kondo K. Tumor-node metastasis staging system for thymic epithelial tumors. $J$ Thorac Oncol. 2010;5(10 Suppl 4):S352-6.

8. Kondo K, Van Schil P, Detterbeck FC, Okumura M, Stratton K, Giroux D, et al. The IASLC/ITMIG thymic epithelial tumors staging project: proposals for the $\mathrm{N}$ and $\mathrm{M}$ components for the forthcoming (8th) edition of the TNM classification of malignant tumors. J Thorac Oncol. 2014;9(9 Suppl 2): S81-7.

9. Omasa M, Date H, Sozu T, Sato T, Nagai K, Yokoi K, et al. Postoperative radiotherapy is effective for thymic carcinoma but not for thymoma in stage II and III thymic epithelial tumors: the Japanese Association for Research on the thymus database study. Cancer. 2015;121:1008-16.

10. Bhora FY, Chen DJ, Detterbeck FC, Asamura H, Falkson C, Filosso PL, et al The ITMIG/IASLC thymic epithelial tumors staging project: a proposed lymph node map for thymic epithelial tumors in the forthcoming 8th edition of the TNM classification of malignant tumors. J Thorac Oncol. 2014;9(9 Suppl 2): S88-96.

11. Rice TW, Ishwaran H, Ferguson MK, Blackstone EH, Goldstraw P. Cancer of the esophagus and esophagogastric junction: an eighth edition staging primer. J Thorac Oncol. 2017;12:36-42.

12. Detterbeck FC, Chansky K, Groome P, Bolejack V, Crowley J, Shemanski L, et al. The IASLC lung cancer staging project: methodology and validation used in the development of proposals for revision of the stage classification of NSCLC in the forthcoming (eighth) edition of the TNM classification of lung cancer. J Thorac Oncol. 2016;11:1433-46.

13. Ruffini E, Fang W, Guerrera F, Huang J, Okumura M, Kim DK, et al. The International Association for the study of lung cancer thymic tumors staging project: the impact of the eighth edition of the Union for International Cancer Control and American Joint Committee on cancer TNM stage classification of thymic tumors J Thorac Oncol. 2020;15:436-47.

14. Ruffini E, Filosso PL, Guerrera F, Lausi P, Lyberis P, Oliaro A. Optimal surgical approach to thymic malignancies: new trends challenging old dogmas. Lung Cancer. 2018;118:161-70.

Key Words: thymus, thymic tumors, staging 